\title{
Una revisión temática de las expectativas de los pacientes frente a las conductas del médico*
}

\section{A Thematic Review of Patient's Expectations Regarding Physician's Behavior}

\author{
Arturo Marroquín Rivera a \\ Médico-cirujano. Estudiante de la Maestría en \\ Epidemiología Clínica, Departamento de Epidemiología \\ Clínica y Bioestadística, Facultad de Medicina, \\ Pontificia Universidad Javeriana, Bogotá, Colombia \\ Mónica Botero Bermúdez \\ Médica-cirujana, Departamento de Epidemiología \\ Clínica y Bioestadística, Facultad de Medicina, \\ Pontificia Universidad Javeriana, Bogotá, Colombia \\ Carlos Gómez-Restrepo \\ Médico psiquiatra. Magíster en Epidemiología Clínica. \\ Profesor titular del Departamento de Epidemiología \\ Clínica y Bioestadística y Psiquiatría y Salud Mental, \\ Facultad de Medicina, Pontificia Universidad \\ Javeriana, Bogotá, Colombia
}

a Autor de correspondencia: marroquin.a@javeriana.edu.co

Cómo citar: Marroquín Rivera A, Botero Bermúdez M, Gómez-Restrepo C. Una revisión temática de las expectativas de los pacientes frente a las conductas del médico. Univ. Med. 2020;61(3). https://doi.org/10.11

144/Javeriana.umed61-3.expe

\section{RESUMEN}

Introducción: Actualmente, la medicina tiende a una atención centrada en el paciente. Esto se debe, no solo a la naturaleza de la profesión, sino también a que, inmersos en un sistema de salud en crisis, la satisfacción del paciente puede traducirse en mejores desenlaces en salud y, por ende, al menos a largo plazo, en ahorros importantes para los sistemas de salud. Objetivo: Llevar a cabo una revisión temática de la literatura que tratará sobre las expectativas del paciente frente al comportamiento del médico durante la atención.

Métodos y materiales: Búsqueda en las bases de datos Pubmed, Lilacs y Embase con términos indexados y libres.

Resultados: Se seleccionaron 46 artículos según los criterios de inclusión establecidos, y a partir de la información encontrada, se crearon 4 dimensiones relacionadas con el constructo de las expectativas: confianza, respeto (autonomía), empatía y comunicación. Finalmente, se sintetizó la información en conformidad con estas categorías.

Conclusiones: Aunque pareciera existir una cantidad importante de investigaciones al respecto, también existe mucha divergencia entre los hallazgos. La mayoría de las veces, las investigaciones tratan dimensiones similares, pero las conclusiones son muy heterogéneas y resaltan las diferencias entre diferentes grupos culturales. En este orden de ideas, sugieren una aproximación local frente a las expectativas de los pacientes. Palabras clave 
prioridad del paciente; comunicación; empatía; confianza; relaciones médico-paciente.

\begin{abstract}
Introduction: Nowadays, medical practice emphasizes towards patient-centered care models, which is consistent with the nature of the medical profession. This model has tremendous advantages, especially in the context of a healthcare system that is in crisis. Considering that patients' satisfaction is an important indicator of quality of care that depends on expectations, its evaluation may lead to a better understanding of factors that can modify the perception of care. Moreover, the evaluation of this factors could improve health outcomes, thus reducing costs in the long run.

Purpose: To make a literature review of the expectations of patients regarding doctors' behavior was performed.

Materials and methods: A search on PubMed, Lilacs and Embase databases was made with $\mathrm{MeSH}$ terms and free terms.

Results: 46 articles met the stablished inclusion criteria. 4 key aspects concerning patients`expectations were defined: Trust, respect (autonomy), empathy and communication.

Conclusions: Even if numerous investigations on this matter have been made, findings are very heterogeneous. Most of the times similar key aspects are explored, however, expectations vary depending on cultural differences and their characteristics. Thus, local investigation is suggested to explore expectations of individuals.

Keywords

patient preference; communication; empathy; trust; physicianpatients relation.
\end{abstract}

\section{Introducción}

Desde antaño, la medicina se ha caracterizado por depender inexorablemente de la interacción humana. Esta profesión caracterizada (en sentido teórico) por su compasión, compromiso y amor por la humanidad ha evolucionado hacia, cada vez más, la resolución de problemas agudos susceptibles de repetirse y ha olvidado la piedra angular de su creación: el paciente (1). No obstante, en la actualidad la atención medica ha hecho esfuerzos por enfocarse una vez más en el paciente y dejar atrás la actitud paternalista y autoritaria de los proveedores en salud, especialmente de los médicos. Esta revolución idiosincrática inició a comienzos del siglo pasado y encontró su auge a finales de este (2). Su objetivo principal es revindicar la importancia de la relación médico-paciente, debido tanto a fundamentos éticos como a cambios biológicos positivos para los pacientes que resultan de una adecuada interacción (3). Así, el mundo, inmerso en la medicina basada en la evidencia, vuelve paulatinamente a la apreciación de las percepciones de los pacientes. Estos cambios se manifiestan oportunamente, puesto que el fortalecimiento de la relación con los pacientes puede modificar cursos clínicos y disminuir o aumentar el número de desenlaces como infecciones, caídas y muertes (4).

Por otro lado, consecuencia de la globalización, la sistematización en búsqueda de la eficiencia y la comercialización del sector de la salud pueden lastimar los cimentos de la confianza (5). Asociado a ello, desarrollos tecnológicos y revoluciones culturales han empoderado al paciente hasta el punto de atribuirle una función fundamental en la toma de decisiones en salud (6). Esta emergente participación yace no solo en la adquisición de conocimiento a través de tecnología, sino en la manifestación individual de autonomía propia de la actualidad. Cada vez más, las libertades individuales y la aparición de instituciones que velan por el respeto de estas estimulan la participación de los individuos en la toma de decisiones (7). La rápida transición se debe a fenómenos como internet, que facilitan el acceso a la información médica y dotan al paciente de conocimiento que le permite ser partícipe de las decisiones que se toman conforme a su salud; pero también complejiza su relación con el proveedor de salud $(8,9)$. Como es de esperarse, esta transición cultural y tecnológica no solo trae consigo beneficios; cambios culturales drásticos y modificaciones de las estructuras previamente establecidas traen consecuencias tanto positivas como negativas, que generalmente requieren adaptaciones solo perceptibles y modificables con el transcurrir del tiempo (10).

En el caso de la relación médico-paciente, se observa, en contraposición a la empatía, que el narcisismo conflictúa a ambas partes e impide el adecuado abordaje terapéutico con consecuencias ocasionalmente deletéreas (3). Estudios teorizan que esta relación debería tratarse más como un alianza entre dos partes, 
pues devela un ímpetu de conciliar opiniones, más que la mera idea de una conexión entre dos partes (11). Esta compleja alianza o, cuando menos, la percepción de esta, la modifican múltiples factores, por ejemplo, el contexto psicosocial del paciente $(4,12)$ o las maneras verbales y no verbales del proveedor de salud (12). La importancia del reconocimiento y del estudio de estos factores se origina en que algunos promueven el entendimiento, la resolución de problemas y los logros terapéuticos, mientras otros truncan el adecuado desarrollo de la interacción (13).

Este gran número de factores se traduce en una complejidad multicausal que, a su vez, se manifiesta como una discrepancia entre las percepciones de los médicos y los pacientes frente al servicio de atención en salud (14). Las dos partes tienen criterios de definición diferentes frente a la calidad, el desempeño y el abordaje correcto de la consulta. Por un lado, el médico tiende a pensar instrumentalmente, concentrándose en los síntomas físicos y la resolución del problema de salud; mientras que el paciente busca un comportamiento afectivo en el cual se aborden sus emociones, a veces buscando una sensación de resolución a corto plazo $(15,16,17)$. De ahí que la importancia de la relación entre los proveedores en salud y los pacientes o, más bien, aquellos que reciben la atención (clientes) se encuentre en la mira de muchas investigaciones. La satisfacción del paciente se ha convertido en símbolo de la calidad del cuidado que, afectada por sus expectativas frente a la consulta, merece una minuciosa evaluación (18). Así, el estudio de las expectativas que tienen los pacientes de su proveedor de salud es fundamental, pues la satisfacción derivada del cumplimento de estas no solo se refleja en menor número de litigaciones, sino también en mejores desenlaces y mejor calidad de vida en los pacientes $(19,20)$.

En ese orden de ideas, y teniendo en cuenta las repercusiones frente a la adherencia del paciente, los desenlaces, la calidad de vida (21) y los posibles costos del sistema, se hace vital estudiar cuáles son estas expectativas y qué factores pueden servir como modificadores, especialmente en el contexto de un sistema de salud en crisis, como es el caso de muchos países de bajos y medianos ingresos, como Colombia (22). Para cumplir este cometido se llevó a cabo una revisión temática y, así, una aproximación al estudio de la perspectiva del paciente, que permita indagar a profundidad cuáles son estas expectativas y qué las modifica.

\section{Metodología}

Se realizó una búsqueda electrónica y sistemática en Pubmed, Embase y LILACS durante febrero del 2019. Se usaron los términos MeSH (o Emtree) y términos libres que incluían patient, motivation, expectation, trust, perception, preferences, physician, doctor patient relationship, empathy, respect, gesture, touch, communication, soft skills, bedside manners y sus términos correspondientes en español. En caso de no existir el termino indexado, se utilizaba el término libre. Adicionalmente, se especificó que no se incluyeran estudios realizados en estudiantes y servicios de enfermería con el conector booleano NOT. Esto debido a que nos interesaba específicamente la perspectiva de los pacientes sobre el comportamiento del médico.

Para la escogencia de las fuentes bibliográficas se consideraron todos los trabajos que cumplieran con los siguientes criterios, ya fueran cualitativos, cuantitativos, primarios, secundarios, longitudinales o transversales:

Trabajos que hablaran específicamente de las expectativas frente a las conductas del médico como individuo (se descartaron trabajos que hablaran exclusivamente de las expectativas de la presentación estética de los médicos, así como de otros aspectos físicos. Esto, porque se pretende hacer hincapié en el comportamiento del médico durante la interacción. También se descartaron aquellos que hablaran exclusivamente de las expectativas frente al sistema de salud, de su aseguradora o de centros de cuidado especializado y de otros profesionales de la salud).

Estudios de satisfacción que evaluaran las preferencias y expectativas de los pacientes de las conductas del médico de manera retrospectiva, 
es decir, que tratarán con la satisfacción de los pacientes una vez se daba la interacción.

Los trabajos debían hablar de las expectativas frente a su médico, excluyendo divisiones por especialidad, enfermedad, condiciones críticas o de riesgo elevado y aquellos que se enfocaran en deseos de fin de vida.

Estudios que evaluaran expectativas o satisfacción frente a esas expectativas definidas y no frente a una institución o un momento particular.

Se incluyeron artículos en español, francés, inglés, alemán, italiano y portugués. No hubo restricción por fecha de publicación. Adicionalmente, cabe aclarar que se descartaron artículos de opinión y resúmenes publicados o expuestos en congresos.

Una vez se contó con todas las fuentes bibliográficas, se procedió con un filtro pareado que tenía en cuenta título y resumen. Dos de los autores realizaron esta fase. Posteriormente, se leyó la totalidad de los artículos seleccionados y se descartaron los que no cumpliesen con los criterios establecidos. Con aquellos que sí cumplieran los criterios se realizó la síntesis de la información.

\section{Resultados y discusión}

Con la búsqueda inicial se obtuvieron 5584, 609 y 2825 resultados de Pubmed, Lilacs y Embase, respectivamente. Estos 9018 artículos se filtraron evaluando el título y resumen hasta obtener 96 . Finalmente, se eliminaron duplicados y aquellos que al leerlos completamente discreparan de los criterios mencionados. Obtuvimos 46 para realizar la síntesis de información.

Así, al leer la totalidad de los resultados, se evaluaron los hallazgos presentes en la literatura abordados a través de métodos cualitativos (principalmente entrevistas semiestructuradas y grupos focales) y cuantitativos (encuestas y escalas), de manera directa (expectativas y prioridades) e indirecta (satisfacción), y lo que el paciente espera de la interacción con el médico, siempre y cuando se refirieran a las expectativas y no a la satisfacción relacionada con un sitio en particular. Durante la revisión, se evidenciaron cinco características principales de los médicos para con los pacientes: la comunicación y las habilidades para transmitir la información, la confianza en el médico, la empatía y el respeto de la autonomía y los deseos del paciente. Dado que al menos una de ellas se incluía en todos los resultados de la búsqueda, se decidió ejecutar la revisión conforme a estas dimensiones. Como aclaración, es importante analizar este fenómeno como un entretejido en el que todas las dimensiones observadas se relacionan entre sí, de manera dinámica con el contexto del paciente, como se observa en la figura 1. Pese a que los pacientes y los médicos suelen entenderlas por separado, todas afectan la percepción, las expectativas y las demás dimensiones. También se mencionan otras características con menor frecuencia, pero son como importantes, según algunos estudios (por lo tanto, se mencionan en una última dimensión).

\section{Figura 1.}

Modelo de la relación entre las expectativas del paciente, las dimensiones y la satisfacción

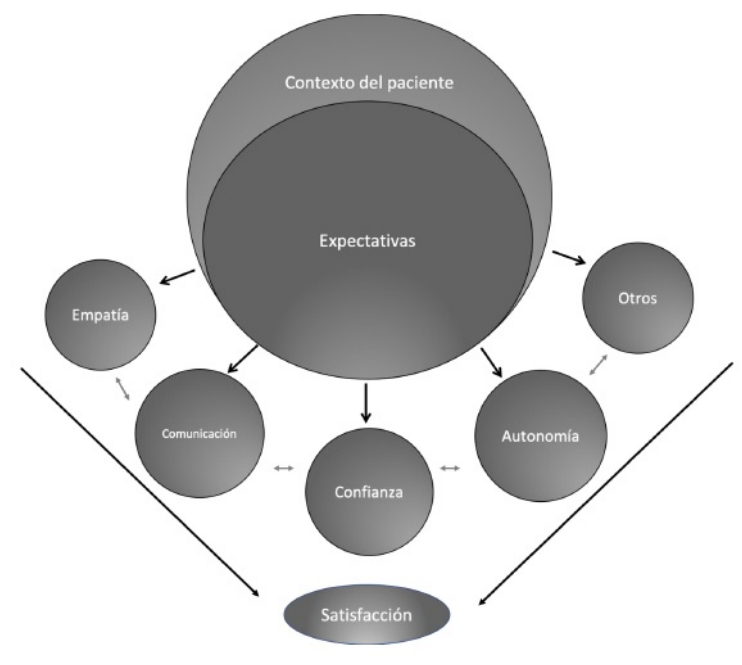

\section{Comunicación efectiva}

La comunicación es considerada la herramienta que permite escuchar, ser escuchado, entender y ser entendido (20). De ahí que el estilo de la comunicación de los médicos generales ha demostrado tener asociaciones importantes con 
la satisfacción del paciente y su adherencia a los manejos terapéuticos (23). La importancia yace en las expectativas, si el médico está al tanto de lo que espera el paciente en términos de profundidad y tipo de información, la comunicación se facilita y aumenta la satisfacción del paciente adulto (24) y adolescente (20).

Independientemente del modelo de cuidado en salud, la comunicación ha demostrado ser un elemento esencial que configura la percepción del paciente sobre la consulta; sin embargo, su caracterización ha resultado compleja por los múltiples elementos que la integran. Para facilitar dicha comprensión, algunos estudios se enfocan en el contenido preverbal; mientras que otros se concentran en las preferencias sobre el contenido de lo comunicado (25). Por otro lado, se ha descrito también un dualismo en cuanto al tipo de comunicación en la relación médicopaciente, a veces calificada de dominante (autoritarismo debido a una asimetría en el conocimiento) o de afectiva (26). El impacto de modificaciones en la comunicación es debatible, ya que el modo deseado de comunicación es también relativo a la cultura y al lenguaje del paciente (24). Por ejemplo, en Corea, aunque un estilo de comunicación dominante generaba más insatisfacción y había más preferencia por comportamientos emocionales y afectivos, la dominancia en la comunicación del médico se relacionaba positivamente con una mayor adherencia $(23,26)$. Otro factor determinante en las expectativas del paciente en cuanto a la comunicación esperada con su médico general es la organización del sistema de salud. Cuando el médico general es la puerta de entrada para ser atendido por algún especialista, no resulta tan importante que sea cuidadoso ni que discuta temas psicosociales. Es decir, la expectativa del paciente concierne en mayor medida al especialista. En cambio, cuando el paciente llega a la consulta sin esperar ser referido a otro, las expectativas recaen en el médico general. Esto se debe a que los pacientes tienden a conocer mejor a su médico y a encontrarse con él más a menudo; por lo tanto, valoran más la comunicación con su médico en las esferas orgánica y psicosocial y, en general, el médico suele prestarse más a brindarla. En el sistema de referencia, los pacientes buscan ser referidos a un psiquiatra si desean discutir temas psicosociales (24). De esta manera, para el estudio de este complejo constructo se han usado tanto métodos cuantitativos como cualitativos, que aun cuando resultan heterogéneos en cuanto a sus enfoques y alcances, concluyen similarmente que para los pacientes lo menos prioritario ante un proceso comunicativo es recibir instrucciones o consejería sobre los comportamientos para mejorar o para prevenir una patología (27).

En un trabajo comparativo acerca de las expectativas frente a la comunicación del médico general en varios países europeos, se encontró que estas variaban significativamente según la cultura del paciente. Otros factores externos que parecen afectar las expectativas de comunicación son la organización del modelo de salud en el que está inmerso el paciente, pues este parece determinar el grado de eficacia y resolución que se espera de un médico general. De manera menos importante también se mencionan el género del paciente, el nivel educativo y el estrato socioeconómico (24). Los pacientes presentan una mayor satisfacción con una mayor comunicación entre los médicos que los trataron durante las hospitalizaciones y aquellos que hacen los controles por fuera de ellas (28). En una revisión sistemática se evidencia que el proveer información es muy importante para los pacientes; sin embargo, en las aproximaciones con métodos cualitativos lo más importante fue la estrategia usada para transmitir información y no la información en sí misma. En la mayoría de los estudios, el estilo comunicativo fue más apreciado y se traducía en mayor satisfacción; no obstante, en Corea se encontró una asociación positiva entre la adherencia y el estilo dominante del médico $(15,24)$. Otro cuestionario basado en las expectativas de los pacientes frente a sus derechos mostró que lo más importante para ellos era el derecho a recibir información sobre la enfermedad, mucho más que el cumplimiento de las regulaciones legales (18). Esta última puede relacionarse con dos dominios: la autonomía y la comunicación. Así, en hospitales de muy alto nivel se ha visto que los pacientes esperan mucho 
profesionalismo: sin embargo, así mismo, esperan ser informados exhaustivamente acerca de su enfermedad (29). Además de la comunicación brindada por el médico, se espera de este tenga una gran capacidad receptiva del lenguaje preverbal del paciente en referencia a sus emociones, sobre todo en los pacientes que se encuentran acostados (30).

Los pacientes priorizan la buena comunicación (31); no les interesa tanto recibir consejos sobre cómo mejorar su estado de salud o la efectividad de su tratamiento, como sí que el médico conozca y les dé importancia a sus problemas psicosociales y se comunique con un lenguaje comprensible al transmitir información (27). Al menos que sea humano en el trato, competente, preciso, que involucre al paciente en las decisiones y explore sus necesidades (31).

Por otro lado, como ya se mencionó, entre los puntos conductuales que afectan la comunicación efectiva se destacan los aspectos preverbales y verbales. Entre los primeros se incluyen que el doctor llame al paciente por el nombre, mantenga contacto visual, salude con un apretón de manos, use un tono de voz suave, confiado y lento, denote comodidad y se presente mediante el título formal y no por el nombre (25). Un aspecto considerado negativo para el paciente es la interrupción con gestos (12). Respecto a las preferencias sobre el contenido de la comunicación se encuentra: que el doctor trate al paciente como un individuo más allá de su enfermedad, que sepa escuchar sin juzgar, que haga preguntas para entender la perspectiva del paciente, que haga uso de su intuición y que con base en todo lo anterior responda a las dudas y preocupaciones del paciente mediante explicaciones comprensibles, es decir, que informe al paciente con la cantidad adecuada de datos y en el nivel necesario $(3,5,14,15,16,17)$. Otras diferencias entre las expectativas de los pacientes frente a la comunicación yacen en el género de quien consulta. Los hombres prefieren que al comunicarse el doctor sea conciso, directo, franco, base sus enunciados en la evidencia y no oculte información sobre el pronóstico del paciente. Algunas mujeres prefieren que el tratante haga énfasis en aspectos psicosociales
(32). Un último factor, poco mencionado pero que cobra relevancia en ciertos contextos, es el humor como herramienta para aliviar la tensión propia de la consulta. Cabe aclarar que este aspecto puede también percibirse como negativo $(32,33)$.

\section{Empatía}

En varios estudios se ha visto que para los pacientes es también prioritario que el doctor sea empático en la interrelación y en la forma de comunicarse $(3,13,14,15,16,17)$, lo cual repercute significativamente en la adherencia y satisfacción de los pacientes, de la mano con otros factores como la percepción que el paciente tenga sobre la experticia del doctor y la habilidad de este último para formar consorcios (23).

El término empatía fue acuñado por Theodor Lipps para referirse a la capacidad de tener apertura a los sentimientos de otra persona. Resalta la necesidad de reconocer la separación entre el yo y lo observado, para no apropiarse del sentir ajeno; en contraste, la simpatía fue entendida como acceder y compartir los sentimientos de otra persona sin identificarlos como ajenos a los propios, lo cual no resultaba resolutivo en la práctica médica $(34,35)$. Así mismo, autores como Truax y Carkhuff, quienes diseñaron una escala para medir el nivel de empatía de los médicos, agregaron que más allá de la sensibilidad al sentimiento ajeno, ser empático implica la facilidad para hacer percibir al otro dicha sensibilidad, en un lenguaje sintonizado con los propios sentimientos (36). Algunos estudios describen también una dualidad teórica en relación con la empatía. Se habla de una naturaleza que puede ser "cognitiva" y que representa la habilidad del médico de percibir el estado mental y sentimental del paciente, o "afectiva", que radica en su sensibilización (26). En pocos estudios es claro a qué se refieren los pacientes al hablar de ella; sin embargo, definen aproximaciones al constructo evaluado.

Teniendo en cuenta lo anterior, la importancia de la empatía radica en permitirle al doctor 
entender el porqué de las acciones de los pacientes y, así, prestarles una atención imparcial en la que sus comportamientos hostiles o demandantes no son motivo de una atención deficiente. De esta manera, se evita la reacciones indeseadas por estos episodios en la conducta del médico y se garantiza una mayor efectividad y un mejor cumplimiento de las metas propuestas (34).

Al indagar sobre este constructo, se encuentran estudios desarrollados en torno al uso de escalas de medición de empatía, entre las que se destacan la Jefferson Scale of Empathy (JCE), el Interpersonal Reactivity Index (IRI), una versión para pacientes de la escala Jefferson (JSPPPE) y la Consultation and Relational Empathy Scale (CARE). Las primeras se enfocan en la percepción de los médicos y el público general sobre su grado de empatía, y las segundas, en evaluar la percepción de los pacientes acerca de la empatía de los doctores. Con estas herramientas se ha demostrado que la percepción de los médicos respecto a su grado de empatía no se correlaciona con la percibida por los pacientes (37). Por otro lado, otros estudios han mostrado que la percepción de una consulta empática ha mostrado mejores desenlaces, medidos en términos de descenso de los valores de la hemoglobina glucosilada y del colesterol malo (LDL) en diabéticos, e incluso un alivio de la sintomatología de los pacientes, visto por ejemplo con un acortamiento de duración de rinofaringitis (38), un mejor control del dolor (39) y una disminución en el informe de los niveles de ansiedad en la consulta. Este último aspecto puede influir también en la percepción del paciente sobre el médico (16). Tanta es la importancia de la empatía para los pacientes, que si se considera empático al médico por medio de su comunicación no verbal, los pacientes lo perciben como cálido y competente, aparte de otras características como el uso de bata. Es decir, la empatía es priorizada por los pacientes, y parece ser un aspecto de suma importancia en comparación con otros aspectos secundarios, como la estética, la infraestructura o el uso de herramientas (35).
Para los pacientes, la empatía se entiende de diversas maneras: se describe como la capacidad del médico de prestarles atención a las emociones negativas del paciente $y$ apaciguarlas (miedo a morir, a estar solo, al abandono, inseguridad, ansiedad, estrés, furia o enajenación) $(3,4,9,15,21)$, como la capacidad de prestar cuidado y cariño al paciente (15) o como un signo de compromiso (38). Así mismo, implica escuchar y entender la perspectiva del paciente (33); saberse comunicar con facilidad, de forma horizontal (sin jerarquías) y resonante, sin recurrir a la actuación $(13,16)$. Por otro lado, otros pacientes consideran que la empatía implica que el médico sea sensible a las inconveniencias generadas por las enfermedades y la hospitalización, y que pueda dar manejo a sus necesidades emocionales y espirituales (40).

Se ha descrito también la percepción de los pacientes sobre los obstáculos que se presentan para prestar una atención empática. Por un lado, mencionan la falta de conexión o solidaridad del médico o, incluso, aspectos como las diferencias entre sus expectativas y las de los doctores. Esto último se debe a que las expectativas pueden afectar la percepción en dos direcciones, tanto por exceso de expectativas como la falta de ellas. Algunos asumen que las expectativas de los pacientes pueden ser demasiado altas y que tienden a enfocarse en el deseo de recibir atención a su esfera psicológica lo que, al no cumplirse, lleva a la decepción. Sin embargo, no tener expectativas también puede generar que el paciente se decepcione de su médico o de la atención brindada. Vale la pena indagar respecto a las expectativas y respecto a lo que puede ser considerado empático.

Finalmente, cabe aclarar que para los pacientes el grado de empatía prestado debe ser individualizado, ya que cada individuo desea involucrar al médico y compartirle un grado distinto de su vida personal y su emocionalidad, sobre todo ante momentos difíciles. Incluso, demasiada empatía puede llevar a que exista un exceso de confianza, de la que algunos pacientes también se arrepienten $(17,41)$. Así, se ha visto que los temas en los que los pacientes más desean intervención empática son en los contextos de 
abuso sexual, de abuso o negligencia por parte de una pareja sentimental y en los intentos de suicidio; mientras que prefieren privacidad en temas referentes a la religión, problemas con el trabajo o desempleo, y moderada intervención en aspectos como el cansancio y problemáticas en la sexualidad (vida sexual) (42).

\section{Confianza}

Un estudio sugiere que medir la confianza puede ser más fidedigno para evaluar la calidad de la relación médico-paciente que medir la satisfacción, e incluso se considera que un modelo de cuidado en salud se construye con base en una relación de confianza $(32,43)$. Así, se ha visto que las experiencias previas del paciente influyen en su grado de confianza, pues pacientes que han tenido disputas previas con los profesionales de la salud tienden a confiar menos en los tratantes, y quienes han decidido abandonar a tratantes previos y buscar nuevos profesionales, tienden a confiar más en ellos. Por otro lado, el nivel de confianza ha mostrado ser proporcional a la cantidad de contacto entre paciente y médico y a qué tanto este último se ajusta a las preferencias del paciente (43).

Por otro lado, llama la atención que aquellos individuos pertenecientes a minorías usualmente registran menores niveles de confianza y satisfacción, aunque no se han visto diferencias con médicos pertenecientes a las mismas minorías, por lo que no se conocen exactamente las causas de este fenómeno (44). Es importante anotar también que la percepción de confianza del paciente puede verse amenazada cuando existe falta de coordinación y consistencia entre los profesionales que prestan la atención (45).

\section{Autonomía}

Para los pacientes, el respeto de su autonomía tiene una enorme relevancia. Esto se entiende como que se tengan en cuenta sus preferencias y su conocimiento a la hora de determinar el diagnóstico y la toma de decisiones en cuanto al tratamiento $(32,41)$. Este deseo, sin embargo, puede verse modificado según el diagnóstico (40).

Así, se cuenta con estudios como el realizado en el 2004 en Hadassah, Jerusalén, en el cual se evidenció que cuatro de los siete atributos preferidos por los pacientes pertenecían al dominio de respeto a la autonomía (31 $\%)$, destacando específicamente el informar de manera adecuada a los pacientes y hacerlos partícipes en las decisiones. Además, se propuso una relación directa entre este dominio y el grado de confianza del paciente, así como un decremento de la satisfacción y aparición de desenlaces adversos al no respetarse su deseo de autonomía. Por otro lado, llama la atención que las características humanísticas — de gran relevancia para los pacientes- no mostraron ser tan prioritarias en este estudio, pues hasta un tercio de los participantes no consideraba importante ninguna característica perteneciente a este dominio (46).

Finalmente, se ha visto que para los padres de pacientes pediátricos el respeto a su autonomía es fundamental, lo cual se traduce en ser informados sobre la situación de su hijo y en que se facilite la comunicación y el intercambio de conocimiento entre los miembros de salud, los cuidadores y los pacientes. Todo esto de la mano de continuidad y consistencia en el servicio (45).

\section{Otras características}

Estas incluyen el que el doctor sea capaz de conocer sus propias limitaciones, que sea competente (evidenciado en su grado de confianza al comunicarse, su habilidad con las tareas físicas y el contenido del conocimiento que administra) $(32,33)$, que tenga profesionalismo (medido en términos de su experticia clínica, actualización del conocimiento, paciencia y enfoque en el paciente) $(46,47)$, que sea respetuoso y que reconozca su responsabilidad ante un error y pida disculpas (41). Cabe aclarar que en uno de los estudios la dimensión más importante para los pacientes hospitalizados fue el profesionalismo, representado por la 
experiencia del médico, aún más importante que el conocimiento percibido (29).

Expectativas de acuerdo con las características particulares de los pacientes

En cuanto al género, algunos estudios evidencian que a la hora de elegir un médico, para los pacientes esta característica no es prioritaria, excepto cuando la consulta trata de afecciones sexuales, situación en la cual prefieren consultar a congéneres $(31,32)$. Por otro lado, en cuanto al contenido de la interacción, se ha visto que los hombres prefieren una comunicación directa, concisa y franca, así como una relación de tipo dominante y consideran prioritario que el médico sea competente, lo que es medible según su manera de actuar, su reputación, su uso del humor y la empatía (32).

De manera similar, se ha visto que la edad del paciente constituye un factor que influye en el grado de satisfacción, por lo que, a mayor edad, más satisfacción, lo cual —se especula- está relacionado con que los adultos mayores tienden a tener mayor respeto a la autoridad y cierta dificultad para exteriorizar sus emociones y quejas. Puede tratarse de un fenómeno generacional (31). Una vez los individuos mayores se expresan, suelen centrarse únicamente en el recibir una atención apresurada o tener que esperar demasiado para ser atendidos, así como recibir explicaciones pobres sobre su situación médica, expresadas mediante un lenguaje complicado o la falta de amabilidad por parte del doctor (33).

Otro factor que influye en las expectativas, en las necesidades y en el deseo de participar en la toma de decisiones o que aborde la emocionalidad es la condición clínica del paciente (40). Se ha visto, por ejemplo, que los pacientes que viven con una discapacidad tienden a considerar prioritario que el médico tenga interés genuino en ellos y en su calidad de vida, que no los juzgue por sus preocupaciones, ni asuma prejuicios sobre sus capacidades, que sea comunicativo y proporcione una atención sin apuros ni interrupciones. También se consideró muy relevante que el médico fuera competente, que se encontrara actualizado y conociera la discapacidad que va a tratar, o en caso de desconocer algún aspecto, fuera capaz de aprender de ella reconociendo la experticia del paciente frente a su propia enfermedad; sin embargo, el aspecto que más pacientes consideraron fundamental fue que el doctor realizara una evaluación holística, en la que se trascendiera la visión del paciente únicamente desde su condición clínica (33).

Por último, se ha visto que el contexto cultural también repercute en las expectativas sobre el abordaje del problema en salud y su tratamiento, al influenciar ideas, creencias y necesidades de los pacientes (24).

\section{Conclusiones}

Aunque la literatura existente sobre las expectativas de los pacientes frente a las conductas de sus médicos es amplia y heterogénea, en la gran mayoría de estudios se resaltó la forma como se presta el servicio, más allá de la competencia técnica, sin que este último sea un factor irrelevante. Otro tipo de aspectos cobran mayor relevancia a la hora de satisfacer las expectativas de los pacientes (15).

Los estudios analizados revelaron que las características más buscadas o esperadas por los pacientes en sus médicos son la capacidad de comunicarse efectivamente, la empatía, la capacidad de generar confianza y el respeto por la autonomía y decisiones de los pacientes. Sin embargo, en la práctica se entiende que el cuidado que cada paciente quiere recibir es subjetivo; por ello, para ajustarse a las expectativas individuales de cada ser humano, el médico debe entender la perspectiva particular de su paciente (41). Lograr este cometido implica considerar factores modificadores de la perspectiva del paciente como el contexto cultural, el género, la edad y el diagnóstico $(24,31)$.

Esta perspectiva del paciente es vital, puesto que a menor discrepancia entre las expectativas del paciente sobre el médico y la atención 
que realmente recibe, mayor satisfacción (15). A su vez, mayor satisfacción se traduce en mejor adherencia, en que el paciente compartirá sus experiencias positivas con otros pacientes $(15,48)$, tenderá a ser más comunicativo y se involucrará más en la consulta $(44,49)$; mientras que un paciente insatisfecho tenderá a desentenderse de las indicaciones médicas y del control con el profesional (15). Por esto, resulta claro que conocer y satisfacer las expectativas del paciente llevan a una mayor adherencia a los tratamientos, a un mejor uso de recursos médicos, a una menor tasa de litigación por mala práctica e, incluso en ocasiones, al alivio de los síntomas $(31,41)$.

De ahí la necesidad de caracterizar las expectativas particulares de los pacientes a nivel local. Por medio de este tipo de investigaciones se puede brindar un servicio de salud de alta calidad, basado en la compresión de las necesidades y expectativas de los pacientes (18).

\section{Referencias}

1. Weir JM, Aicken MD, Cupples ME, Steele K. From hippocrates to the Francis report - Reflections on empathy. Ulster Med J. 2015;84(1):8-12.

2. Armstrong D. The patient's view. Soc Sci Med. 1984;18(9):737-44.

3. Lejoyeux M. The doctor-patient relationship: New psychological models. Bull Acad Natl Med. 2011;195(7):1477-90.

4. MacAllister L, Zimring C, Ryherd E. Environmental variables that influence patient satisfaction: a review of the literature. Heal Environ Res Des J. 2016 Oct;10(1):155-69.

5. Jeffrey D. Empathy, sympathy and compassion in healthcare: Is there a problem? Is there a difference? Does it matter? J R Soc Med [Internet]. 2016 Dec [citado 2019 feb 12];109(12):446-52. Disponible en: https://journals.sagepub.com/doi/p df/10.1177/0141076816680120

6. Wisniewski JM, Diana ML, Yeager VA, Hotchkiss DR. Comparison of objective measures and patients' perceptions of quality of services in government health facilities in the Democratic Republic of Congo. Int J Qual Heal Care. 2018 Jul;30(6):472-9.

7. Hamelin ND, Nikolis A, Armano J, Harris PG, Brutus JP. Evaluation of factors influencing confidence and trust in the patient-physician relationship: A survey of patient in a hand clinic. Chir Main. 2012;31(2):83-90.

8. Tustin N. The role of patient satisfaction in online health information seeking. J Health Commun. 2010;15(1):3-17.

9. Olakowski M, Hładoń A, Seweryn M, Ciosek J, Świątkiewicz W. Patients' Opinions about Polish Surgeons and Surgical Treatment. Pol Przegl Chir. 2016;88(5):238-44.

10. Bensing JM, Rimondini M, Fletcher I, van Vliet L, Van Bogaert M, Moretti $F$, et al. How to make the medical consultation more successful from a patient's perspective? Tips for doctors and patients from lay people in the United Kingdom, Italy, Belgium and the Netherlands. Patient Educ Couns. 2011 Sep;84(3):287-93.

11. Fuertes JN, Toporovsky A, Reyes M, Osborne JB. The physician-patient working alliance: Theory, research, and future possibilities. Patient Educ Couns [Internet]. $2017 \mathrm{Apr} ; 100(4): 610-5$. htt ps://doi.org/10.1016/j.pec.2016.10.018

12. Little P, White P, Kelly J, Everitt H, Gashi S, Bikker A, et al. Verbal and non-verbal behaviour and patient perception of communication in primary care: An observational 
study. Br J Gen Pract. 2015 Jun 25;65(635):e357-65.

13. Gómez G, Aillach E. Ways to improve the patient-physician relationship. Curr Opin Psychiatry. 2013;26(5):453-7.

14. Hermans L, Olde Hartman T, Dielissen PW. Differences between GP perception of delivered empathy and patient-perceived empathy: a crosssectional study in primary care. $\mathrm{Br} \mathrm{J}$ Gen Pract. 2018;68(674):e621-6.

15. Miaoulis GJ, Gutman J, Snow MM. Closing the gap: the patientphysician disconnect. Health Mark Q. 2009;26(1):56-68.

16. van Dulmen S, van den BrinkMuinen A. Patients' preferences and experiences in handling emotions: a study on communication sequences in primary care medical visits. Patient Educ Couns. 2004 Oct;55(1):149-52.

17. Derksen FAWM, Hartman TO, Bensing J, Lagro-Janssen A. Empathy in general practice-the gap between wishes and reality: Comparing the views of patients and physicians. Fam Pract. 2018;35(2):203-8.

18. Bostan S, Acuner T, Yilmaz GGG. Patient (customer) expectations in hospitals. Health Policy [Internet]. 2007 Jun;82 (1):62-70. https://doi.org/1 0.1016/j.healthpol.2006.08.005

19. Cubaka VK, Schriver M, Kayitare JB, Cotton P, Maindal HT, Nyirazinyoye L, et al. "He should feel your pain": Patient insights on patient-provider communication in Rwanda. African J Prim Heal Care Fam Med. 2018;10(1). 20. Daley AM, Polifroni EC, Sadler LS. "Treat me like a normal person!" A Meta-ethnography of adolescents' expectations of their health care providers. J Pediatr Nurs. 2017;36:70-83.
21. Rozenblum R, Lisby M, Hockey PM, Levtizion-Korach O, Salzberg CA, Lipsitz S, et al. Uncovering the blind spot of patient satisfaction: An international survey. BMJ Qual Saf. 2011;20(11):959-65. https://doi.org/10 $.1136 /$ bmjqs-2011-000306

22. Iragorri Amaya N, Triana Romero PA. Metodología del análisis de costo-efectividad (ACE) en la práctica clínica. Investig Segur Soc Salud [internet]. 2015;17(1):67-76. Disponible en: https://pesquisa.bvsalud .org/portal/resource/pt/biblio-966022

23. Kim SS, Kaplowitz S, Johnston M V. The effects of physician empathy on patient satisfaction and compliance. Eval Health Prof. 2004 Sep;27(3):237-51.

24. van den Brink-Muinen $A$, Verhaak PFM, Bensing JM, Bahrs O, Deveugele M, Gask L, et al. Doctor-patient communication in different European health care systems: Relevance and performance from the patients' perspective. Patient Educ Couns. 2000;39(1):115-27.

25. Davis RL, Wiggins MN, Mercado CC, O'Sullivan PS. Defining the core competency of professionalism based on the patient's perception. Clin Experiment Ophthalmol. 2007;35(1):51-4.

26. Kim SS, Park BK. Patient-perceived communication styles of physicians in rehabilitation: the effect on patient satisfaction and compliance in Korea. Am J Phys Med Rehabil. 2008 Dec;87 (12):998-1005.

27. Deledda G, Moretti F, Rimondini M, Zimmermann C. How patients want their doctor to communicate. A literature review on primary care patients' perspective. Patient Educ Couns. 2013;90(3):297-306. 
28. Adams DR, Flores A, Coltri A, Meltzer DO, Arora VM. A missed opportunity to improve patient satisfaction? Patient perceptions of inpatient communication with their primary care physician. Am J Med Qual. 2016 Nov;31(6):568-76.

29. Schattner A, Rudin D, Jellin N. Good physicians from the perspective of their patients. BMC Health Serv Res [Internet]. 2004 Sep [citado 2019 Mar 18];4(1):26. Disponible en: http://www .biomedcentral.com/1472-6963/4/26

30. Mazzi MA, Bensing J, Rimondini M, Fletcher I, van Vliet L, Zimmermann C, et al. How do lay people assess the quality of physicians' communicative responses to patients' emotional cues and concerns? An international multicentre study based on videotaped medical consultations. Patient Educ Couns. 2013 Mar;90(3):347-53.

31. Wensing M, Jung HP, Mainz J, Olesen F, Grol R. A systematic review of the literature on patient priorities for general practice care. Part 1: Description of the research domain. Soc Sci Med. 1998 Nov;47 (10):1573-88.

32. Smith JA, Braunack-Mayer AJ, Wittert GA, Warin MJ. Qualities men value when communicating with general practitioners: implications for primary care settings. Med J Aust. 2008 Dec;189(11-12):618-21.

33. Shapiro J, Mosqueda L, Botros D. A caring partnership: expectations of ageing persons with disabilities for their primary care doctors. Fam Pract. 2003 Dec;20(6):635-41.

34. Aring CD. Sympathy and empathy. J Am Med Assoc. 1958 May;167(4):448-52.

35. Kraft-Todd GT, Reinero DA, Kelley JM, Heberlein AS, Baer L, Riess H. Empathic nonverbal behavior increases ratings of both warmth and competence in a medical context. PLoS One. 2017;12(5):e0177758.

36. Poole AD, Sanson-Fisher RW. Understanding the patient: a neglected aspect of medical eduction. Soc Sci Med Med Psychol Med Sociol. 1979 Jan;13A(1):37-43.

37. Bernardo MO, Cecílio-Fernandes D, Costa P, Quince TA, Costa MJ, Carvalho-Filho MA, et al. Physicians' self-assessed empathy levels do not correlate with patients' assessments. PLoS One. 2018;13(5). https://doi.org/ 10.1371/journal.pone.0198488

38. Derksen F, Hartman TCO, Van Dijk A, Plouvier A, Bensing J, LagroJanssen A. Consequences of the presence and absence of empathy during consultations in primary care: $\mathrm{A}$ focus group study with patients. Patient Educ Couns. 2017;100:987-93. http:// dx.doi.org/10.1016/j.pec.2016.12.003

39. Mercer SW, Reilly D, Watt GCM. The importance of empathy in the enablement of patients attending the Glasgow Homoeopathic Hospital. Br J Gen Pract. 2002 Nov;52(484):901-5.

40. Gesell SB, Wolosin RJ. Inpatients' ratings of care in 5 common clinical conditions. Qual Manag Health Care. 2004;13(4):222-7.

41. Quirk M, Mazor K, Haley H-L, Philbin M, Fischer M, Sullivan K, et al. How patients perceive a doctor's caring attitude. Patient Educ Couns. 2008 Sep;72(3):359-66.

42. Frowick B, Shank JC, Doherty WJ, Powell TA. What do patients really want? Redefining a behavioral science curriculum for family physicians. J Fam Pract. 1986 Aug;23(2):141-6.

43. Balkrishnan R, Dugan E, Camacho FT, Hall MA. Trust and satisfaction with physicians, insurers, and the 
medical profession. Med Care. 2003 Sep;41(9):1058-64.

44. Paez KA, Allen JK, Beach MC, Carson KA, Cooper LA. Physician cultural competence and patient ratings of the patient-physician relationship. J Gen Intern Med. 2009 Apr;24(4):495-8.

45. Heller KS, Solomon MZ. Continuity of care and caring: what matters to parents of children with lifethreatening conditions. J Pediatr Nurs. 2005 Oct;20(5):335-46.

46. Schattner A, Rudin D, Jellin N. Good physicians from the perspective of their patients. BMC Health Serv Res. 2004 Sep;4(1):26.

47. Wiggins MN, Coker K, Hicks EK. Patient perceptions of professionalism: implications for residency education. Med Educ. 2009 Jan;43(1):28-33.

48. Elleuch A. Patient satisfaction in Japan. Int J Health Care Qual Assur. 2008;21(7):692-705.

49. Street RLJ, Gordon H, Haidet P. Physicians' communication and perceptions of patients: is it how they look, how they talk, or is it just the doctor? Soc Sci Med. 2007 Aug;65(3):586-98.

\section{Notas}

* Este trabajo no recibió ningún tipo de financiación. 